\title{
Impact of antihypertensive treatment on left ventricular systolic dyssynchrony in treatment-naïve hypertensive patients
}

\author{
Beom-June Kwon, Sung-Won Jang, Kyu-Young Choi, Dong-Bin Kim, Eun-Joo Cho, Sang-Hyun Ihm, \\ Ho-Joong Youn, Tai-Ho Rho and Jae-Hyung Kim
}

Dyssynchrony is common in asymptomatic patients with hypertension. We sought to investigate the impact of antihypertensive treatment on dyssynchrony in patients with hypertension. A total of sixty patients who had uncomplicated hypertension that had never been treated (treatment-naïve hypertensive patients) underwent echocardiographic evaluations of left ventricular (LV) dyssynchrony at baseline and after a 6-month treatment with antihypertensive drugs. The measured parameters were as follows: (1) the s.d. of 12 LV-segment time-to-peak systolic velocities (Ts-SD12), and (2) the maximal difference between peak systolic velocities of any 2 of the 12 segments (Ts-Max). Patients with Ts-SD12 $\geqslant 33 \mathrm{~ms}$ or Ts-Max $\geqslant 100 \mathrm{~ms}$ were regarded as having LV systolic dyssynchrony. Patients with systolic dyssynchrony (group $1, n=29$ ) and without systolic dyssynchrony (group 2 , $n=31$ ) were compared. Among the patients in group 1, antihypertensive treatment significantly improved LV systolic dyssynchrony ( $\Delta$ Ts-SD12, $-13.1 \mathrm{~ms} ; \boldsymbol{P}<0.001$ and $\Delta$ Ts-Max, $-34.0 \mathrm{~ms} ; \boldsymbol{P}=0.003$ ), whereas it did not demonstrate additional benefit among group 2 patients. The change in LV systolic dyssynchrony was significantly associated with changes in the mean annulus $E^{\prime}$ velocity, mean annulus $S^{\prime}$ velocity and mean annulus $E^{\prime} / A^{\prime}$ ratio, but not with changes in blood pressure and $L V$ mass index. It is likely that chronic antihypertensive treatment could reverse the LV systolic dyssynchrony and simultaneously improve subclinical systolic and diastolic function in patients with hypertension and LV systolic dyssynchrony.

Hypertension Research (2012) 35, 661-666; doi:10.1038/hr.2012.28; published online 15 March 2012

Keywords: antihypertensive treatment; diastole; Doppler; dyssynchrony; echocardiography; systole

\section{INTRODUCTION}

It is well known that left ventricular (LV) systolic or diastolic dyssynchrony is common in patients with heart failure (HF) and a normal QRS duration. ${ }^{1}$ There has been increased recognition that LV dyssynchrony also exists in patients without HF but with other cardiac diseases, including hypertension, ${ }^{2}$ coronary artery disease $(\mathrm{CAD})^{3}$ and cardiomyopathy. ${ }^{4,5}$ Dyssynchrony has been measured using a variety of echocardiographic parameters based on different imaging techniques, ${ }^{6}$ including pulsed-wave tissue Doppler imaging (TDI), colorcoded TDI, tissue tracking, displacement mapping, strain and strain rate imaging, and tissue synchronization imaging. TDI is the most extensively tested method.

Hypertension is a major risk factor for LV hypertrophy and HF. Epidemiological studies have demonstrated that hypertension accounts for $50 \%$ of patients with $\mathrm{HF}^{7}$ Recently, it was demonstrated that LV systolic dyssynchrony was present in $24 \%$ of asymptomatic patients with hypertension. ${ }^{2}$ Moreover, on the basis of results from the previous study, ${ }^{8}$ the proportion of treatment-naïve hypertensives with LV systolic dyssynchrony was found to be $44.5 \%$.
Research has indicated that antihypertensive treatment reverses LV remodeling and LV hypertrophy in patients with hypertension; ${ }^{9}$ however, the impact of antihypertensive treatment on dyssynchrony has rarely been determined. We therefore designed a prospective study to identify the impact of antihypertensive treatment on dyssynchrony in patients who had uncomplicated hypertension that had never been treated.

\section{METHODS}

Study population

We consecutively enrolled patients who were diagnosed with primary hypertension between January 2009 and February 2010 at St Paul's Hospital (The Catholic University of Korea, Seoul, Korea). None of the patients had ever received antihypertensive treatment before they visited our hospital.

Hypertension was diagnosed based on clinical blood pressure (BP) measurements $\geqslant 140 / 90 \mathrm{~mm} \mathrm{Hg}$ on two separate occasions. Inclusion criteria were as follows: (1) outpatients of either gender, $18-75$ years of age; (2) mild to moderate hypertension (diastolic BP $<110 \mathrm{mmHg}$ and systolic BP $<180 \mathrm{~mm} \mathrm{Hg}$ ); and (3) a normal QRS duration $(<120 \mathrm{~ms})$. Exclusion criteria 
were as follows: (1) previous use of antihypertensive medications; (2) secondary hypertension; (3) diabetes mellitus, defined as a fasting plasma glucose $\geqslant 126 \mathrm{mg} \mathrm{dl}^{-1}$ or a history of diabetes mellitus; (4) renal insufficiency, defined as a serum creatinine $\geqslant 1.5 \mathrm{mg} \mathrm{dl}^{-1}$; (5) CAD; (6) HF, according to the Framingham criteria or a low LV ejection fraction $(<50 \%)$; (7) cerebrovascular disease or peripheral artery disease; (8) echocardiographic evidence of valvular heart disease, $\mathrm{LV}$ regional wall motion abnormalities, pulmonary hypertension, or pericardial disease, or inaccurate delimination of the endocardial border; and (9) rhythm disorders, including atrioventricular block, incomplete or complete bundle branch block, pre-excitation syndrome, ventricular arrhythmias or atrial fibrillation.

A treadmill exercise test, 24-h ECG monitoring, cardiac single photon emission computed tomography, coronary computed tomography and/or coronary angiography were performed if the patient was suspected to have ischemic heart disease. Body mass index was calculated as the body weight $(\mathrm{kg})$ divided by the height $(\mathrm{m})$ squared. Metabolic syndrome was defined according to the International Diabetes Federation criteria. ${ }^{10}$ Estimated glomerular filtration rate was calculated by the Modification of Diet in Renal Disease formula. ${ }^{11}$

The study protocol was approved by the Institutional Review Board for Biomedical Research at our institution. Written informed consent was obtained from all subjects.

\section{Antihypertensive treatment}

Antihypertensive treatment was initiated after baseline echocardiography was performed. The angiotensin II receptor blocker (ARB) is one of the first-line antihypertensive drugs for most patients, but monotherapy achieves the target BP recommended by the treatment guidelines ${ }^{12,13}$ in only a limited number of patients and, thus, combination therapy is required in a majority of patients. ${ }^{12}$ A thiazide diuretic is commonly used in combination with an ARB because it has an additive effect on BP reduction because of its complementary mechanisms of action. ${ }^{12}$ Patients first received monotherapy with ARB (Telmisartan, up to $80 \mathrm{mg})$. When the target $\mathrm{BP}(<140 / 90 \mathrm{~mm} \mathrm{Hg})$ was not reached within 4 weeks, we added hydrochlorothiazide (up to $50 \mathrm{mg}$ ) to the treatment regimen. Patients were followed up every 4 weeks thereafter. If the patient's clinical BP was greater than $140 / 90 \mathrm{~mm} \mathrm{Hg}$ or intolerance to drugs occurred, a $\beta$-blocker (Cavedilol, up to $50 \mathrm{mg}$ ) was added. Finally, a calcium antogonist (Nifedipine, up to $60 \mathrm{mg}$ ) was added. The dose of medication could be downtitrated to the previous level in the event of hypotension, dizziness or intolerance. Once patients reached the target BP, they were maintained on the dose of antihypertensive drugs at which the target $\mathrm{BP}$ was reached for the study duration. Patients who could not obey this protocol were dropped from the study. Patients were followed up for 6 months.

\section{Echocardiographic assessment}

Standard TDI and strain echocardiography were performed with a commercially available echocardiography system (Vivid 7; GE Vingmed Ultrasound AS, Horten, Norway). Standard two-dimensional and color Doppler data (three consecutive beats), triggered to the QRS complex, were saved in a cine loop format. The averages of at least three consecutive beats were used for comparison, and all data were analyzed offline using a dedicated software package (EchoPAC version 6.0.1, GE Healthcare, Milwaukee, WI, USA) for TDI and TDI-derived parameters. All echocardiographic measurements were performed by one physician who was blinded to the clinical data to avoid interobserver variation. Echocardiography was performed at baseline and 6 months after antihypertensive therapy.

Standard echocardiography. The following parameters were evaluated, including Doppler and TDI studies: (1) the LV volumes and ejection fraction were assessed by biplane Simpson rule using manual tracing of digital images of the apical 4- and 2-chamber views according to the American Society of Echocardiography standard; ${ }^{14}$ (2) LV diameters were measured using twodimensional-guided M-mode echocardiography according to the guidelines of the American Society of Echocardiography; ${ }^{15}$ and (3) transmitral flow velocities were recorded from the apical 4-chamber view using pulse-wave Doppler. The peak flow velocity in early diastole (E) and late diastole (A), the E/A ratio, the deceleration time of $\mathrm{E}$-wave and the isovolumic relaxation time were obtained.
The filling time was measured from the onset of the E-wave to the end of the A-wave. Pulsed-TDI of the four sides of the mitral annulus (septal, lateral, inferior and anterior) was also obtained in 4- and 2-chamber views to assess global cardiac function. ${ }^{16,17}$ (4) The subaortic flow velocity was obtained by pulsed-wave Doppler from the apical 5-chamber view. The LV ejection time was measured as the interval between the onset of forward aortic flow and the aortic valve closure artifact.

Dyssynchrony-related echocardiography.

(1) LV systolic dyssynchrony: time-to-peak systolic velocity (Ts) and the standard deviation (s.d.) of $12 \mathrm{LV}$ segments (Ts-SD12) were calculated with reference to the QRS complex. ${ }^{1}$ Tissue Doppler time-velocity curves were derived from the following 12 sites: septal, lateral, anterior, inferior, anteroseptal and posterior at both basal and mid-levels from the apical 4-, 3- and 2-chambers. The maximal difference between peak systolic velocities of any 2 of the 12 segments (Ts-Max) was also calculated. ${ }^{18}$ The time delay between Ts at the LV basal lateral and septal segments (Ts-LS) was measured. ${ }^{19,20}$ A Ts-SD12 $\geqslant 33$ or Ts-Max $\geqslant 100 \mathrm{~ms}$ was regarded as the presence of LV systolic dyssynchrony. ${ }^{21}$

(2) LV diastolic dyssynchrony: the time-to-peak myocardial early diastolic velocity ( $\mathrm{Te}$ ) of the $12 \mathrm{LV}$ segments were measured with reference to the QRS complex and the s.d. of Te of all $12 \mathrm{LV}$ segments (Te-SD12), and the maximal difference in Te between any 2 of the $12 \mathrm{LV}$ segments (Te-Max) was calculated. A Te-SD12 $\geqslant 34$ or Te-Max $\geqslant 113 \mathrm{~ms}$ was regarded as the presence of diastolic dyssynchrony. ${ }^{21}$

(3) Contractile diastolic dyssynchrony: the sum of the time of strain tracing exceeding aortic valve closure on the overall $12 \mathrm{LV}$ basal and midsegments (oExcT) was measured. ${ }^{22}$

(4) Each parameter was obtained from three consecutive cardiac cycles and was only included in the algorithm for $<10 \%$ beat-to-beat variation to improve reproducibility and accuracy. Intraobserver variability was calculated as the mean absolute difference in LV systolic dyssynchrony measurements divided by the original measurements and expressed as a percentage. The intraobserver variabilities of Ts-SD12, Ts-Max, Te-SD12, Te-Max and oExcT were found to be $6.8 \%, 7.0 \%, 6.5 \%, 6.6 \%$ and $5.9 \%$, respectively.

\section{Statistical analyses}

On the basis of the results of the preliminary data comparing hypertensive patients with and without LV systolic dyssynchrony, we assumed 25\% (derived from a one-half difference between two groups) improvement of Ts-SD12 in the group with LV systolic dyssynchrony and no interval change in the group without LV systolic dyssynchrony. Calculation of the sample size was based on a two-sided alpha level of 0.05 and $80 \%$ power. The total sample size was estimated to be 60 patients (1:1 assigned per group) for the trial on the expectation of $10 \%$ patient loss during echocardiography follow-up. The baseline characteristics of the patients were analyzed according to the presence or absence of LV systolic dyssynchrony. Continuous variables were expressed as the mean \pm s.d. and compared using an unpaired $t$-test. Categorical variables were expressed as a number (percentage) and compared using a $\chi^{2}$-test or Fisher's exact test (if there was an expected cell value $<5$ ). Changes of clinical and echocardiographic measurements before and after antihypertensive therapy were compared with paired $t$-tests. Linear associations between two variables were measured by Pearson's correlation coefficient $(r)$ with additional adjustments for potential confounders. Analysis of covariance was performed to compare the parameters between the two groups after adjustments for potential confounders, which were $P<0.20$ in the univariate analysis, age and gender. Statistical significance was set to $P<0.05$. All analyses were conducted using SAS 9.1 (SAS Institute, Cary, NC, USA).

\section{RESULTS}

\section{Patient and baseline clinical characteristics}

A total of 78 patients fulfilling the inclusion and exclusion criteria were initially evaluated and underwent echocardiographic examination. Of those, 18 were excluded because the images were not suitable for 
Table 1 Baseline clinical characteristics of the study population

\begin{tabular}{|c|c|c|c|}
\hline & $\begin{array}{c}\text { HT with LV systolic dyssynchrony } \\
\text { (Group 1, } \mathrm{n}=29 \text { ) }\end{array}$ & $\begin{array}{l}\text { HT without LV systolic dyssynchrony } \\
\text { (Group 2, } \mathrm{n}=31 \text { ) }\end{array}$ & P-values \\
\hline Age (years) & $53 \pm 9$ & $55 \pm 10$ & 0.439 \\
\hline Body mass index $\left(\mathrm{kg} \mathrm{m}^{-2}\right)$ & $26.0 \pm 2.8$ & $25.0 \pm 3.2$ & 0.175 \\
\hline $\mathrm{SBP}(\mathrm{mm} \mathrm{Hg})$ at office & $162 \pm 17$ & $157 \pm 17$ & 0.264 \\
\hline DBP $(\mathrm{mm} \mathrm{Hg})$ at office & $98 \pm 14$ & $96 \pm 11$ & 0.521 \\
\hline Fasting plasma glucose $\left(\mathrm{mg} \mathrm{dl}^{-1}\right)$ & $110 \pm 11$ & $108 \pm 12$ & 0.905 \\
\hline Hyperlipidemia, $n$ (\%) & $13(44.8)$ & $22(71.0)$ & 0.040 \\
\hline Metabolic syndrome, $n(\%)$ & $15(51.7)$ & $18(41.9)$ & 0.448 \\
\hline Estimated GFR by MDRD formula ( $\mathrm{ml} \mathrm{min}^{-1}$ per $1.73 \mathrm{~m}^{2}$ ) & $84.5 \pm 16.6$ & $81.5 \pm 11.7$ & 0.413 \\
\hline Current smoker, $n(\%)$ & $3(10.3)$ & $12(38.7)$ & 0.011 \\
\hline $\mathrm{Hs}-\mathrm{CRP}\left(\mathrm{mg} \mathrm{dl}^{-1}\right)$ & $0.22 \pm 0.20$ & $0.23 \pm 0.18$ & 0.786 \\
\hline
\end{tabular}

Abbreviations: DBP, diastolic blood pressure; GFR, glomerular filtration rate; Hs-CRP, high-sensitive C-reactive protein; HT, hypertension; LV, left ventricular; MDRD, Modification of Diet in Renal Disease Study Group; $n$, number; SBP, systolic blood pressure.

Values are arithmetic mean \pm s.d.

Table 2 Classes of medical treatment in the hypertensive patients

\begin{tabular}{|c|c|c|c|}
\hline \multicolumn{4}{|l|}{ Anti-hypertensive treatment } \\
\hline Thiazide, $n(\%)$ & $22(75.9)$ & $20(64.5)$ & 0.338 \\
\hline$\beta$-blockers, $n(\%)$ & $12(41.4)$ & $9(29.0)$ & 0.316 \\
\hline Calcium antagonists, $n(\%)$ & $1(3.4)$ & $0(0.0)$ & 0.297 \\
\hline
\end{tabular}

Abbreviations: ARB, angiotensin receptor blocker; HT, hypertension; LV, left ventricular; $n$, number; target blood pressure (BP) goal, $<140 / 90 \mathrm{~mm} \mathrm{Hg}$.

Values are arithmetic mean \pm s.d.

evaluating dyssynchrony parameters. Of the 60 patients enrolled, 29 revealed LV systolic dyssynchrony (group 1), whereas the others did not (group 2). Among the patients with LV systolic dyssynchrony, the proportion who had a Ts-SD $12 \geqslant 33 \mathrm{~ms}$ was $100 \%$ ( 29 of 29 patients), and the proportion who had a Ts-Max $\geqslant 100 \mathrm{~ms}$ was $82.8 \%$ (24 of 29 patients). Follow-up echocardiography was performed after a 6-month antihypertensive treatment.

The baseline clinical characteristics of the patients are summarized in Table 1. Patients in group 1 experienced less hyperlipidemia and represented fewer current smokers than those in group 2 .

\section{Antihypertensive treatment}

There were no significant differences in the classes of medications, numbers of drugs or percentage of achieving a target BP goal $(<140 /$ $90 \mathrm{~mm} \mathrm{Hg}$ ) between the two groups (Table 2).

\section{Baseline echocardiographic measurements}

The parameters of conventional echocardiography at baseline were not significantly different between groups 1 and 2 (Table 3). Both groups demonstrated normal systolic and diastolic function at baseline. However, as expected, patients in group 1 had severe LV systolic dyssynchrony (Ts-SD12, $P<0.001$; Ts-Max, $P<0.001$; and Ts-LS, $P<0.001)$ compared with the group 2 patients (Table 4 ).
Changes in BP and conventional echocardiograpic measurements after antihypertensive treatment

After 6 months of antihypertensive treatment, all of the patients experienced significant improvements in systolic BP, diastolic BP, the mean annulus $\mathrm{E}^{\prime}$ and the mean annulus $\mathrm{E}^{\prime} / \mathrm{A}^{\prime}$ ratio compared with baseline (Table 3 ). In addition, the group 1 patients demonstrated significant improvements in the LV mass index (LVMI), mitral E/A ratio and mean annulus $S^{\prime}$ after antihypertensive treatment, compared with baseline.

\section{Changes in dyssynchrony parameters of echocardiograpy after 6} months of antihypertensive treatment

Table 4 summarizes the changes in the LV dyssynchrony parameters after antihypertensive treatment. Antihypertensive treatment significantly improved LV systolic dyssynchrony for the group 1, but not the group 2, patients. After adjustment for age, gender, body mass index, hyperlipidemia and current smoking status, the improvement of LV dyssynchrony among group 1 patients remained significant (Ts-SD12, $P<0.001$; Ts-Max, $P=0.004$; and Ts-LS, $P<0.001$ ). However, the values of Ts-SD12 and Ts-Max after antihypertensive treatment in group 1 patients were significantly greater than those of the group 2 patients (Ts-SD12, $P=0.014$; Ts-Max, $P=0.024$; and Ts-LS, $P=0.015$ ). In addition, global, atrioventricular and interventricular dyssynchrony 
Table 3 Effects of antihypertensive treatment on clinical characteristics and standard echocardiography in the hypertensive patients

\begin{tabular}{|c|c|c|c|c|c|c|c|c|}
\hline & \multicolumn{4}{|c|}{$H T$ with LV systolic dyssynchrony (Group 1, $\mathrm{n}=29$ ) } & \multicolumn{4}{|c|}{$H T$ without $L V$ systolic dyssynchrony (Group 2, $\mathrm{n}=31$ ) } \\
\hline & Baseline & $\begin{array}{c}\text { After } \\
6 \text { months }\end{array}$ & $\begin{array}{l}\Delta \text { change } \\
(95 \% \mathrm{Cl})\end{array}$ & $\begin{array}{c}\text { P-values } \\
\text { (paired t-test) }\end{array}$ & Baseline & $\begin{array}{c}\text { After } \\
6 \text { months }\end{array}$ & $\begin{array}{l}\Delta \text { change } \\
(95 \% \mathrm{Cl})\end{array}$ & $\begin{array}{c}\text { P-values } \\
\text { (paired } t \text {-test) }\end{array}$ \\
\hline SBP $(\mathrm{mm} \mathrm{Hg})$ at office & $162 \pm 17$ & $128 \pm 11$ & $-34(-39$ to -28$)$ & $<0.001$ & $157 \pm 17$ & $130 \pm 10$ & $-27(-34$ to -19$)$ & $<0.001$ \\
\hline $\mathrm{DBP}(\mathrm{mm} \mathrm{Hg})$ at office & $98 \pm 14$ & $80 \pm 6$ & $-18(-23$ to -14$)$ & $<0.001$ & $96 \pm 11$ & $83 \pm 9$ & $-13(-18$ to -9$)$ & $<0.001$ \\
\hline QRS duration (ms) & $85 \pm 15$ & $82 \pm 13$ & $-3(-8$ to 3$)$ & 0.250 & $86 \pm 13$ & $84 \pm 13$ & -2 ( -7 to 3$)$ & 0.323 \\
\hline \multicolumn{9}{|l|}{ LV size and function } \\
\hline Ejection fraction (\%) & $64.7 \pm 5.6$ & $66.1 \pm 7.2$ & $1.4(-1.3$ to 4.1$)$ & 0.294 & $64.4 \pm 7.5$ & $63.6 \pm 7.5$ & $-0.7(-4.2$ to 2.7$)$ & 0.672 \\
\hline End-diastolic volume (ml) & $68.7 \pm 18.9$ & $66.0 \pm 17.1$ & $-2.7(-9.1$ to 3.7$)$ & 0.397 & $64.3 \pm 20.0$ & $63.4 \pm 17.9$ & $-0.9(-7.3$ to 5.6$)$ & 0.786 \\
\hline End-systolic volume (ml) & $24.5 \pm 8.4$ & $22.6 \pm 7.9$ & $-1.9(-5.4$ to 1.5$)$ & 0.262 & $23.5 \pm 10.5$ & $22.8 \pm 7.7$ & $-0.7(-4.5$ to 3.0$)$ & 0.698 \\
\hline LV mass index $\left(\mathrm{g} \mathrm{m}^{-2}\right)$ & $102.5 \pm 33.1$ & $90.4 \pm 24.2$ & $-12.1(-21.8$ to -2.3$)$ & 0.017 & $106.0 \pm 30.3$ & $94.6 \pm 25.7$ & $-11.4(-23.0$ to 0.1$)$ & 0.052 \\
\hline Mitral E wave $\left(\mathrm{cm} \mathrm{s}^{-1}\right)$ & $74.4 \pm 18.1$ & $76.8 \pm 19.3$ & $0.12(0.02$ to 0.22$)$ & 0.339 & $66.5 \pm 14.6$ & $68.2 \pm 16.6$ & $0.07(-0.02$ to 0.15$)$ & 0.551 \\
\hline Mean annulus $\mathrm{E}^{\prime}\left(\mathrm{cm} \mathrm{s}^{-1}\right)$ & $7.5 \pm 2.3$ & $8.6 \pm 2.6$ & 0.09 (0.01 to 0.17 ) & $<0.001$ & $7.4 \pm 2.5$ & $8.2 \pm 2.5$ & 0.08 (0.02 to 0.13 ) & $<0.001$ \\
\hline Mean annulus $\mathrm{A}^{\prime}\left(\mathrm{cm} \mathrm{s}^{-1}\right)$ & $10.2 \pm 1.6$ & $10.3 \pm 1.4$ & $1.4(-1.3$ to 4.1$)$ & 0.670 & $9.5 \pm 1.4$ & $9.6 \pm 1.3$ & $-0.7(-4.2$ to 2.7$)$ & 0.833 \\
\hline Mean annulus $E^{\prime} / A^{\prime}$ ratio & $0.76 \pm 0.26$ & $0.85 \pm 0.28$ & $-2.7(-9.1$ to 3.7$)$ & 0.021 & $0.80 \pm 0.31$ & $0.87 \pm 0.32$ & $-0.9(-7.3$ to 5.6$)$ & 0.005 \\
\hline
\end{tabular}

Abbreviations: $\mathrm{Cl}$, confidence interval; DBP, diastolic blood pressure; HT, hypertension; LV, left ventricular; Mean annulus A', mean myocardial late diastolic velocity of four annulus of LV segments; Mean annulus $E^{\prime}$, mean myocardial early diastolic velocity of four annulus of LV ventricular segments; Mean annulus $\mathrm{S}^{\prime}$, mean myocardial systolic velocity of four annulus of LV segments; Mitral E/A, peak early diastolic mitral flow velocity/peak late diastolic mitral flow velocity; $n$, number; SBP, systolic blood pressure; $\Delta$, value of follow-up minus value of baseline. Values are arithmetic mean \pm s.d.

Table 4 Effects of antihypertensive treatment on dyssynchrony in the hypertensive patients

\begin{tabular}{|c|c|c|c|c|c|c|c|c|}
\hline & \multicolumn{4}{|c|}{ HT with LV systolic dyssynchrony (Group $1, \mathrm{n}=29$ ) } & \multicolumn{4}{|c|}{ HT without LV systolic dyssynchrony (Group 2, $\mathrm{n}=31$ ) } \\
\hline & Baseline & $\begin{array}{c}\text { After } \\
6 \text { months }\end{array}$ & $\Delta$ change $(95 \% \mathrm{Cl})$ & $\begin{array}{c}\text { P-values } \\
\text { (paired t-test) }\end{array}$ & Baseline & After 6 months & $\Delta$ change $(95 \% \mathrm{Cl})$ & $\begin{array}{c}\text { P-values } \\
\text { (paired t-test) }\end{array}$ \\
\hline \multicolumn{9}{|c|}{ LV systolic dyssynchrony } \\
\hline Ts-SD12 (ms) & $48.6 \pm 11.1^{*}$ & $35.6 \pm 16.5 \dagger$ & $-13.1(-19.8$ to -6.4$) \dagger \dagger$ & $<0.001$ & $23.1 \pm 8.3$ & $25.0 \pm 15.7$ & $1.9(-5.3$ to 9.1$)$ & 0.592 \\
\hline Ts-Max (ms) & $133.4 \pm 38.2^{*}$ & $99.4 \pm 43.6 \dagger$ & $-34.0(-55.2$ to -12.9$) \dagger \dagger$ & 0.003 & $99.4 \pm 27.3$ & $72.7 \pm 45.1$ & $4.7(-16.8$ to 26.1$)$ & 0.659 \\
\hline Ts-LS (ms) & $89.4 \pm 38.4^{*}$ & $57.7 \pm 89.4 \dagger$ & $-31.6(-50.9$ to -12.4$) \dagger \dagger$ & 0.002 & $21.8 \pm 23.9$ & $32.5 \pm 29.3$ & $10.7(-3.5$ to 24.9$)$ & 0.134 \\
\hline \multicolumn{9}{|c|}{ LV diastolic dyssynchrony } \\
\hline Te-SD12 (ms) & $21.0 \pm 8.1$ & $19.8 \pm 6.7$ & $-1.2(-4.1$ to 1.7$)$ & 0.414 & $24.0 \pm 12.9$ & $23.7 \pm 12.2$ & $-0.3(-5.1$ to 4.5$)$ & 0.893 \\
\hline Te-Max (ms) & $69.5 \pm 26.1$ & $61.2 \pm 19.5$ & $-8.3(-18.3$ to 1.8$)$ & 0.102 & $74.7 \pm 36.8$ & $74.4 \pm 37.7$ & $-0.3(-14.2$ to 13.5$)$ & 0.960 \\
\hline \multicolumn{9}{|c|}{ Contractile diastolic dyssynchrony } \\
\hline oExcT (ms) & $559.7 \pm 329.4$ & $431.0 \pm 208.7$ & $-128.7(-275.4$ to 18.0$)$ & 0.083 & $526.8 \pm 305.4$ & $400.4 \pm 162.5$ & $-126.4(-243.8$ to -9.0$)$ & 0.036 \\
\hline
\end{tabular}

between two groups were not different before and after antihypertensive treatment (Supplementary Tables 1 and 2).

\section{Relationships between changes in LV systolic dyssynchrony and other parameters}

Linear relationships between changes in LV systolic dyssynchrony and other parameters that demonstrated significant changes after medical treatment were analyzed after adjustment for potential confounding factors, including age, gender, body mass index, fasting plasma glucose, hyperlipidemia, estimated glomerular filtration rate, current smoking status and change in heart rate (Table 5). The changes in LV systolic dyssynchrony (Ts-SD12 and Ts-Max) were significantly associated with changes in the mean annulus $\mathrm{E}^{\prime}$ velocity, the mean annulus $\mathrm{S}^{\prime}$ velocity and the mean annulus $\mathrm{E}^{\prime} / \mathrm{A}^{\prime}$ ratio, but not with $\mathrm{BP}$ and LVMI. In particular, mean annulus velocity was associated with the severity of LV systolic dyssynchrony at baseline (Supplementary Table 3). 
Table 5 Linear correlations between changes of LV systolic dyssynchrony ( $\Delta$ Ts-SD12 or $\Delta$ Ts-Max) and other parameters in all hypertensive patients

\begin{tabular}{|c|c|c|}
\hline Variables & $R$ & P-values \\
\hline \multicolumn{3}{|l|}{ Mode/a for $\Delta T s-S D 12$} \\
\hline$\Delta \mathrm{SBP}(\mathrm{mm} \mathrm{Hg})$ at office & 0.246 & 0.079 \\
\hline$\triangle \mathrm{DBP}(\mathrm{mm} \mathrm{Hg})$ at office & 0.198 & 0.160 \\
\hline$\Delta \mathrm{LV}$ mass index $\left(\mathrm{g} \mathrm{m}^{-2}\right)$ & 0.069 & 0.627 \\
\hline$\Delta$ Mitral E/A ratio & -0.108 & 0.446 \\
\hline$\Delta$ Mean annulus $\mathrm{E}^{\prime}\left(\mathrm{cm} \mathrm{s}^{-1}\right)$ & -0.340 & 0.014 \\
\hline$\Delta$ Mean annulus $\mathrm{S}^{\prime}\left(\mathrm{cm} \mathrm{s}^{-1}\right)$ & -0.308 & 0.026 \\
\hline$\Delta$ Mean annulus $E^{\prime} / A^{\prime}$ ratio & -0.289 & 0.038 \\
\hline \multicolumn{3}{|l|}{ Mode/ ${ }^{\text {a }}$ for $\Delta T s$-Max } \\
\hline$\Delta \mathrm{SBP}(\mathrm{mm} \mathrm{Hg})$ at office & 0.241 & 0.085 \\
\hline$\triangle \mathrm{DBP}(\mathrm{mm} \mathrm{Hg})$ at office & 0.264 & 0.058 \\
\hline$\Delta \mathrm{LV}$ mass index $\left(\mathrm{g} \mathrm{m}^{-2}\right)$ & 0.051 & 0.719 \\
\hline$\Delta$ Mitral E/A ratio & -0.051 & 0.718 \\
\hline$\Delta$ Mean annulus $E^{\prime}\left(\mathrm{cm} \mathrm{s}^{-1}\right)$ & -0.347 & 0.012 \\
\hline$\Delta$ Mean annulus $\mathrm{S}^{\prime}\left(\mathrm{cm} \mathrm{s}^{-1}\right)$ & -0.278 & 0.046 \\
\hline$\Delta$ Mean annulus $E^{\prime} / A^{\prime}$ ratio & -0.271 & 0.052 \\
\hline
\end{tabular}

Abbreviations: DBP, diastolic blood pressure; $\Delta$, value of follow-up minus value of baseline; LV, left ventricular; Mean annulus $A^{\prime}$, mean myocardial late diastolic velocity of four annulus of LV segments; Mean annulus $E^{\prime}$, mean myocardial early diastolic velocity of four annulus of LV ventricular segments; Mean annulus $\mathrm{S}^{\prime}$, mean myocardial systolic velocity of four annulus of $L V$ segments; SBP, systolic blood pressure.

${ }^{a}$ Adjusted for age, sex, body mass index, fasting plasma glucose, hyperlipidemia, estimated glomerular filtration rate, current smoker and change of heart rate.

\section{DISCUSSION}

In this trial of patients with uncomplicated primary hypertension that had never been treated, antihypertensive treatment significantly reversed LV systolic dyssynchrony. The benefit was observed among patients with LV systolic dyssynchrony at baseline, but not among patients without LV systolic dyssynchrony. After adjustment for confounding factors, medical treatment in patients with LV systolic dyssynchrony was also beneficial. In addition, the reversal of LV systolic dyssynchrony was significantly and independently associated with an improvement in subclinical systolic (annulus $\mathrm{S}^{\prime}$ velocity) and diastolic (annulus $\mathrm{E}^{\prime}$ velocity and annulus $\mathrm{E}^{\prime} / \mathrm{A}^{\prime}$ ratio) function. Our results, together with those of a previous study, ${ }^{23}$ provide evidence that a change in LV systolic dyssynchrony is associated with a transmitral flow pattern or annulus velocity. Our study population is representative of patients with new onset hypertension. All patients demonstrated narrow QRS complexes without HF and were naïve to antihypertensive medications.

Previous trials have focused on patients with structural heart disease, including $\mathrm{CAD},{ }^{3}$ hypertrophic cardiomyopahthy, ${ }^{5}$ dilated cadiomyopathy ${ }^{4}$ and subsequent HF. ${ }^{1} \mathrm{LV}$ dyssynchrony is known to impair the function of a failing ventricle, resulting in the worsening of symptoms and clinical outcomes in patients with HF. ${ }^{24,25}$ LV dyssynchrony is associated with the development of LV remodeling after acute myocardial infarction ${ }^{26}$ and increases the risk of new-onset HF in patients with hypertension. ${ }^{27}$ The effects of medical treatment on dyssynchrony in patients with $\mathrm{CAD},{ }^{28}$ hypertrophic cardiomyopahthy, ${ }^{29}$ dilated cadiomyopathy ${ }^{30}$ and $\mathrm{HF}^{30-32}$ have been reported, but patients with hypertension and a structurally normal heart have not been evaluated. Our trial demonstrated the benefit of chronic antihypertensive treatment on dyssynchrony in patients with early stage hypertension. These findings emphasize the potential importance of dyssynchrony as a pharmacological target and predictor of adverse clinical events. Specifically in early stage hypertension, dyssynchrony measurement may be a parameter for pharmacological interference on the transition from uncomplicated hypertension to HF. A longer-term study will be required to determine whether changes of LV dyssynchrony in hypertension are associated with the subsequent development of HF. The clinical implications and impacts of LV systolic dyssynchrony on morbidities and prognosis still remain unclear in hypertensive patients.

In this trial, the LVMI was significantly decreased after antihypertensive treatment. It is well known that angiotensin-converting enzyme inhibitors, ARBs and $\beta$-blockers, can reverse LV remodeling and LV hypertrophy. ${ }^{9,33}$ Our study lends further support to the notion that antihypertensive treatment can reduce LV hypertrophy; however, there was no significant relationship between the LVMI and the LV systolic dyssynchrony, which is in accordance with the results from a previous trial. ${ }^{34}$ The benefits of antihypertensive treatment on LV systolic dyssynchrony may be independent of LVMI regression. In contrast, Yang et al. $^{35}$ demonstrated the association between LV systolic dyssynchrony and LV mass. Several factors could explain this discrepancy. First, in a study by Yang et al., 24\% of the patients had diabetes, and all of the patients had already been taking antihypertensive medication, whereas we included only treatmentnaïve hypertensives and non-diabetics. Second, although there were many confounding factors, including cardiac status, comorbidities and medications, they only performed univariate analysis; the significance may be changed in a multivariate analysis.

The high severity and prevalence of dyssynchrony in treatmentnaive patients with uncomplicated hypertension ${ }^{8}$ may be explained, at least in part, by myocardial fibrosis, ${ }^{36}$ exaggerated accumulation of collagen fibers, ${ }^{37}$ altered myocardial glucose metabolism, ${ }^{38}$ amplified transmural repolarization dispersion caused by LV hypertrophy ${ }^{39}$ and long-term cardiac memory. ${ }^{40}$ Therefore, the mechanisms by which medical treatment could lead to the reversal of LV systolic dyssynchrony can be explained by substantial improvements in the abovementioned causes. In addition, the BP-lowering effect and subsequent reduction of afterload may have an important role.

Some regional contractile events can be shifted in the diastolic phase. Mechanical dyssynchrony in the systolic phase has been an important issue, but delayed LV contractions in the diastolic phase after aortic valve closure have not received focus. Contractile diastolic dyssynchrony can occur independently of delayed activation in various conditions, such as ischemia, LV hypertrophy or LV overload. ${ }^{41}$ The finding of an improvement in contractile diastolic dyssynchrony demonstrated that antihypertensive treatment may have a benefit in the diastolic as well as the systolic phase, in terms of longitudinal contraction. Further clinical investigations are needed to determine the real clinical impact of an improvement in contractile diastolic dyssynchrony.

The present study had some limitations. First, the study population was relatively small, and whether the subjects of this study represented the general population was not established. Second, we used the most popular TDI method to measure LV dyssynchrony, but there is no gold standard method for detecting mechanical dyssynchrony. ${ }^{42}$ Third, it is unclear whether our results were dependent on the escalation protocol of the antihypertensive drugs. Finally, it is uncertain whether the improvement of LV systolic dyssynchrony by antihypertensive treatment could be maintained beyond 6 months.

In conclusion, the present study confirmed that chronic antihypertensive treatment significantly reverses LV systolic dyssynchrony and simultaneously improves subclinical systolic and diastolic function in treatment-naïve hypertensive patients. Further studies are warranted to determine whether improvements in dyssynchrony prevent HF and adverse cardiovascular events. 
1 Yu CM, Lin H, Zhang Q, Sanderson JE. High prevalence of left ventricular systolic and diastolic asynchrony in patients with congestive heart failure and normal QRS duration. Heart 2003; 89: 54-60.

2 Chang SA, Kim H, Kim D, Kim Y, Sohn D, Oh B, Park Y. Left ventricular systolic and diastolic dyssynchrony in asymptomatic hypertensive patients. J Am Soc Echocardiogr 2009; 22: 337-342.

3 Yu C, Yang H, Lau C, Wang Q, Wang S, Lam L, Sanderson JE. Regional left ventricle mechanical asynchrony in patients with heart disease and normal QRS duration: implication for biventricular pacing therapy. Pacing Clin Electrophysiol 2003; 26 562-570.

4 Schuster I, Habib G, Jego C, Thuny F, Avierinos J, Derumeaux G, Beck L, Medail C, Franceschi F, Renard S, Ferracci A, Lefevre J, Luccioni R, Deharo J, Djiane P. Diastolic asynchrony is more frequent than systolic asynchrony in dilated cardiomyopathy and is less improved by cardiac resynchronization therapy. J Am Coll Cardiol 2005; 46: 2250-2257.

5 Nagakura T, Takeuchi M, Yoshitani H, Nakai H, Nishikage T, Kokumai M, Otani S, Yoshiyama M, Yoshikawa J. Hypertrophic cardiomyopathy is associated with more severe left ventricular dyssynchrony than is hypertensive left ventricular hypertrophy. Echocardiography 2007; 24: 677-684.

6 Gorcsan J, Abraham T, Agler DA, Bax J, Derumeaux G, Grimm RA, Martin R, Steinberg JS, Sutton MS, Yu C. Echocardiography for cardiac resynchronization therapy: recommendations for performance and reporting - a report from the American society of echocardiography dyssynchrony writing group endorsed by the heart rhythm society. J Am Soc Echocardiogr 2008; 21: 191-213.

7 Levy D, Larson MG, Vasan RS, Kannel WB, Ho KK. The progression from hypertension to congestive heart failure. JAMA 1996; 275: 1557-1562.

8 Kwon BJ, Choi KY, Kim DB, Jang SW, Cho EJ, Youn HJ, Kim JH. Systolic synchrony is impaired in nonleft ventricular hypertrophy of never-treated hypertensive patients. J Hypertens 2011; 29: 2246-2254.

9 Devereux RB, Dahlof B, Gerdts E, Boman K, Nieminen MS, Papademetriou V, Rokkedal J, Harris KE, Edelman JM, Wachtell K. Regression of hypertensive left ventricular hypertrophy by losartan compared with atenolol: the Losartan Intervention for Endpoint Reduction in Hypertension (LIFE) trial. Circulation 2004; 110: 1456-1462.

10 Alberti KG, Zimmet P, Shaw J. The metabolic syndrome-a new worldwide definition. Lancet 2005; 366: 1059-1062.

11 Levey AS, Bosch JP, Lewis JB, Greene T, Rogers N, Roth D. A more accurate method to estimate glomerular filtration rate from serum creatinine: a new prediction equation. Ann Intern Med 1999; 130: 461-470.

12 Mancia G, De Backer G, Dominiczak A, Cifkova R, Fagard R, Germano G, Grassi G, Heagerty AM, Kjeldsen SE, Laurent S, Narkiewicz K, Ruilope L, Rynkiewicz A, Schmieder RE, Boudier HA, Zanchetti A, Vahanian A, Camm J, De Caterina R, Dean V, Dickstein K, Filippatos G, Funck-Brentano C, Hellemans I, Kristensen SD, McGregor K, Sechtem U, Silber S, Tendera M, Widimsky P, Zamorano JL, Erdine S, Kiowski W, Agabiti-Rosei E, Ambrosioni E, Lindholm LH, Viigimaa M, Adamopoulos S, Bertomeu V, Clement D, Farsang C, Gaita D, Lip G, Mallion JM, Manolis AJ, Nilsson PM, O'Brien E, Ponikowski P, Redon J, Ruschitzka F, Tamargo J, van Zwieten P, Waeber B, Williams B. 2007 Guidelines for the management of arterial hypertension: the task force for the management of arterial hypertension of the European Society of Hypertension (ESH) and of the European Society of Cardiology (ESC). J Hypertens 2007; 25: 1105-1187.

13 Chobanian AV, Bakris GL, Black HR, Cushman WC, Green LA, Izzo Jr JL, Jones DW, Materson BJ, Oparil S, Wright Jr JT, Roccella EJ. Seventh report of the joint national committee on prevention, detection, evaluation, and treatment of high blood pressure. Hypertension 2003; 42: 1206-1252.

14 Lang RM, Bierig M, Devereux RB, Flachskampf FA, Foster E, Pellikka PA, Picard MH, Roman MJ, Seward J, Shanewise JS, Solomon SD, Spencer KT, Sutton MS, Stewart WJ. Recommendations for chamber quantification: a report from the American society of echocardiography's guidelines and standards committee and the chamber quantification writing group, developed in conjunction with the European association of Echocardiography, a branch of the European society of cardiology. J Am Soc Echocardiogr 2005; 18: 1440-1463

15 Sahn DJ, DeMaria A, Kisslo J, Weyman A. Recommendations regarding quantitation in M-mode echocardiography: results of a survey of echocardiographic measurements. Circulation 1978; 58: 1072-1083.

16 Wang M, Yip G, Yu CM, Zhang Q, Zhang Y, Tse D, Kong SL, Sanderson JE. Independent and incremental prognostic value of early mitral annulus velocity in patients with impaired left ventricular systolic function. J Am Coll Cardiol 2005; 45: 272-277.

17 Alam M, Wardell J, Andersson E, Samad BA, Nordlander R. Characteristics of mitral and tricuspid annular velocities determined by pulsed wave Doppler tissue imaging in healthy subjects. J Am Soc Echocardiogr 1999; 12: 618-628.

18 Agler DA, Adams DB, Waggoner AD. Cardiac resynchronization therapy and the emerging role of echocardiography (part 2): the comprehensive examination. J Am Soc Echocardiogr 2007; 20: 76-90.

19 Bax J, Marwick TH, Molhoek SG, Bleeker GB, van Erven L, Boersma E, Steendijk P, van der Wall E, Schalij MJ. Left ventricular dyssynchrony predicts benefit of cardiac resynchronization therapy in patients with end-stage heart failure before pacemaker implantation. Am J Cardiol 2003; 92: 1238-1240.
20 Bax J, Molhoek SG, van Erven L, Voogd PJ, Somer S, Boersma E, Steendijk P, Schalij MJ, Van der Wall E. Usefulness of myocardial tissue Doppler echocardiography to evaluate left ventricular dyssynchrony before and after biventricular pacing in patients with idiopathic dilated cardiomyopathy. Am J Cardiol 2003; 91: 94-97.

21 Yu C, Zhang Q, Yip GW, Lee P, Kum LC, Lam Y, Fung JW. Diastolic and systolic asynchrony in patients with diastolic heart failure: a common but ignored condition. J Am Coll Cardiol 2007; 49: 97-105.

22 Porciani MC, Lilli A, Macioce R, Cappelli F, Demarchi G, Pappone A, Ricciardi G, Padeletti L. Utility of a new left ventricular asynchrony index as a predictor of reverse remodelling after cardiac resynchronization therapy. Eur Heart $J$ 2006; 27: 1818-1823.

23 Ciampi Q, Petruzziello B, Della Porta M, Caputo S, Manganiello V, Astarita C, Villari B. Effect of intraventricular dyssynchrony on diastolic function and exercise tolerance in patients with heart failure. Eur J Echocardiogr 2009; 10: 907-913.

24 Duncan AM, Francis DP, Gibson DG, Henein MY. Limitation of exercise tolerance in chronic heart failure: distinct effects of left bundle-branch block and coronary artery disease. J Am Coll Cardiol 2004; 43: 1524-1531.

25 Baldasseroni S, Opasich C, Gorini M, Lucci D, Marchionni N, Marini M, Campana C, Perini G, Deorsola A, Masotti G, Tavazzi L, Maggioni AP. Left bundle-branch block is associated with increased 1 -year sudden and total mortality rate in 5517 outpatients with congestive heart failure: a report from the Italian network on congestive heart failure. Am Heart J 2002; 143: 398-405.

26 Mollema SA, Bleeker GB, Liem SS, Boersma E, van der Hoeven BL, Holman ER, van der Wall EE, Schalij MJ, Bax JJ. Does left ventricular dyssynchrony immediately after acute myocardial infarction result in left ventricular dilatation? Heart Rhythm 2007; 4: 1144-1148.

27 Okin PM, Devereux RB, Kjeldsen SE, Edelman JM, Dahlof B. Incidence of heart failure in relation to QRS duration during antihypertensive therapy: the LIFE study. J Hypertens 2009; 27: 2271-2277

28 Nii T, Nakashima Y, Kawano T, Arakawa K. Reduced left ventricular diastolic asynchrony after captopril in single vessel coronary artery disease. Cardiology 1992; 81: 115-120.

29 Emoto R, Yokota Y, Miki T, Usuki S, Nomura H, Takeuchi Y, Nakatani M, Fukuzaki H. Effects of verapamil on the left ventricular diastolic function and diastolic asynchrony in patients with hypertrophic cardiomyopathy. J Cardiol 1991; 21: 87-96.

30 Takemoto Y, Hozumi T, Sugioka K, Takagi Y, Matsumura Y, Yoshiyama M, Abraham TP, Yoshikawa J. Beta-blocker therapy induces ventricular resynchronization in dilated cardiomyopathy with narrow QRS complex. J Am Coll Cardiol 2007; 49: 778-783.

31 Mullens W, Borowski AG, Curtin R, Grimm RA, Thomas JD, Tang WH. Mechanical dyssynchrony in advanced decompensated heart failure: relation to hemodynamic responses to intensive medical therapy. Heart Rhythm 2008; 5: 1105-1110.

32 Wang J, Kurrelmeyer KM, Torre-Amione G, Nagueh SF. Systolic and diastolic dyssynchrony in patients with diastolic heart failure and the effect of medical therapy. J Am Coll Cardiol 2007; 49: 88-96.

33 Mathew J, Sleight P, Lonn E, Johnstone D, Pogue J, Yi Q, Bosch J, Sussex B, Probstfield J, Yusuf S. Reduction of cardiovascular risk by regression of electrocardiographic markers of left ventricular hypertrophy by the angiotensin-converting enzyme inhibitor ramipril. Circulation 2001; 104: 1615-1621.

34 Bae BS, Kim KJ, Park JG, Jung YS, Ryu HJ, Kang HJ, Lee BR, Jung BC. Improvement in left ventricular systolic dyssynchrony in hypertensive patients after treatment of hypertension. Korean Circ J 2011; 41: 16-22.

35 Yang B, Chettiveettil D, Jones F, Aguero M, Lewis JF. Left ventricular dyssynchrony in hypertensive patients without congestive heart failure. Clin Cardiol 2008; 31: 597-601.

36 Kai H, Kuwahara F, Tokuda K, Imaizumi T. Diastolic dysfunction in hypertensive hearts: roles of perivascular inflammation and reactive myocardial fibrosis. Hypertens Res 2005; 28: 483-490.

37 Dez J, Gonzlez A, Lpez B, Querejeta R. Mechanisms of disease: pathologic structural remodeling is more than adaptive hypertrophy in hypertensive heart disease. Nat Clin Pract: Cardiovasc Med 2005; 2: 209-216.

38 Matsumoto K, Takahashi N, Ishikawa T, Sumita S, Matsushita K, Inoue N, Kobayashi T, Uchino K, Kimura K, Inoue T, Umemura S. Evaluation of myocardial glucose metabolism before and after recovery of myocardial function in patients with tachycardiainduced cardiomyopathy. Pacing Clin Electrophysiol 2006; 29: 175-180.

39 Yan GX, Rials SJ, Wu Y, Liu T, Xu X, Marinchak RA, Kowey PR. Ventricular hypertrophy amplifies transmural repolarization dispersion and induces early after depolarization. Am J Physiol Heart Circ Physiol 2001; 281: H1968-H1975.

40 Patberg KW, Rosen MR. Molecular determinants of cardiac memory and their regulation. J Mol Cell Cardiol 2004; 36: 195-204.

41 Citro R, Galderisi M. Myocardial postsystolic motion in ischemic and not ischemic myocardium: the clinical value of tissue Doppler. Echocardiography 2005; 22: 525-532.

42 Chung ES, Leon AR, Tavazzi L, Sun J, Nihoyannopoulos P, Merlino J, Abraham WT, Ghio S, Leclercq C, Bax J, Yu C, Gorcsan J, St John Sutton M, De Sutter J, Murillo J. Results of the Predictors of Response to CRT (PROSPECT) trial. Circulation 2008; 117: 2608-2616.

Supplementary Information accompanies the paper on Hypertension Research website (http://www.nature.com/hr) 